\title{
Lessons Learned During the Transition of SORCE Science Operations to Daylight Only Operations
}

\author{
Sean Ryan, ${ }^{1}$ Emily Pilinski, ${ }^{2}$ and Deb McCabe ${ }^{3}$ \\ Laboratory for Atmospheric and Space Physics, Boulder CO, 80303, USA
}

In July 2013, NASA's Solar Radiation and Climate Experiment experienced a battery anomaly which placed it into safemode halting all science observations. Initial attempts to recover the spacecraft to an operational configuration failed due to the reduced capacity of the battery. As the keystone mission for measuring total solar irradiance, and the cornerstone mission for measuring the solar spectral irradiance there was a strong motivation for developing a new operations concept that would allow SORCE to resume daily measurements of the Sun. The operations team faced many challenges over the next several months. For a fiveday period in late 2013 the operations team was able resume science observations to cross-calibrate SORCE data with a new instrument launched in November 2013. After the cross-calibration campaign was completed a new operations concept was deployed which allowed SORCE to perform daylight only operations. In this mode of operations all non-essential components are powered off at each eclipse entry and then turned back on at sunrise. In March 2014 SORCE resumed making daily measurements of the Sun. This paper will review the events and lessons learned from the six-month recovery effort.

\section{SORCE Mission}

In 1999, NASA selected the Laboratory for Atmospheric and Space Physics (LASP) at the University of Colorado to manage, develop, and operate a dedicated spacecraft to study solar irradiance. The mission is a Principal Investigator-led mission with program management performed by LASP with oversight from NASA's Goddard Space Flight Center (GSFC). Originally designed as a five-year mission, NASA's Solar Radiation and Climate Experiment (SORCE) was launched into low Earth orbit in January 2003 and carries with it four instruments to measure the incoming solar radiation at the top of the Earth's atmosphere. The mission is an important component of NASA's Earth Observing System. The scientific goals of the SORCE are to make daily measurements of the Total Solar Irradiance (TSI) and of Spectral Solar Irradiance (SSI) from 120 to $2000 \mathrm{~nm}$. Solar radiation is the dominant energy source in the solar system [1] and understanding of its variation is critical for atmospheric and climate studies. The TSI and SSI records are essential components of the national climate program with the continuous TSI data record going back to the Earth Radiation Budget instrument in 1979. Figure 1 contains a plot of the aggregate TSI measurements for the last 35 years from several different instruments [2]. As can be seen, there are significant differences in the TSI measured between early instruments.

LASP partnered with Goddard Space Flight Center and Orbital Sciences Corporation (OSC), now Orbital ATK, to fully implement the mission. OSC was responsible for providing the spacecraft bus, launch vehicle and leading the integration and testing of the fully assembled observatory. LASP was responsible for the development of the instruments, mission operations, science data processing and overall program management.

The importance of continuing the SORCE TSI measurement increased in March of 2011 when the first absolutely calibrated TSI instrument, Glory-Total Irradiance Monitor (TIM), was lost in a launch vehicle failure. Due to measurement offsets seen in Figure 1, the continuity of measurements from one TSI instrument to the next is important to the scientific community. As a result, it was important to continue the SORCE observations until such a time where its measurements could be compared to another absolutely calibrated TSI instrument. In order to reduce the likelihood of a gap in the TSI measurements, NASA funded the TSI Calibration Transfer Mission (TCTE). Unfortunately, at the time of the SORCE battery anomaly the TCTE instrument was still several months from launch.

\footnotetext{
${ }^{1}$ SORCE Mission Operations Manager, Mission Operations and Data Systems Group.

${ }^{2}$ SORCE Flight Controller, Mission Operations and Data Systems Group.

${ }^{3}$ SORCE Flight Director, Mission Operations and Data Systems Group.
} 


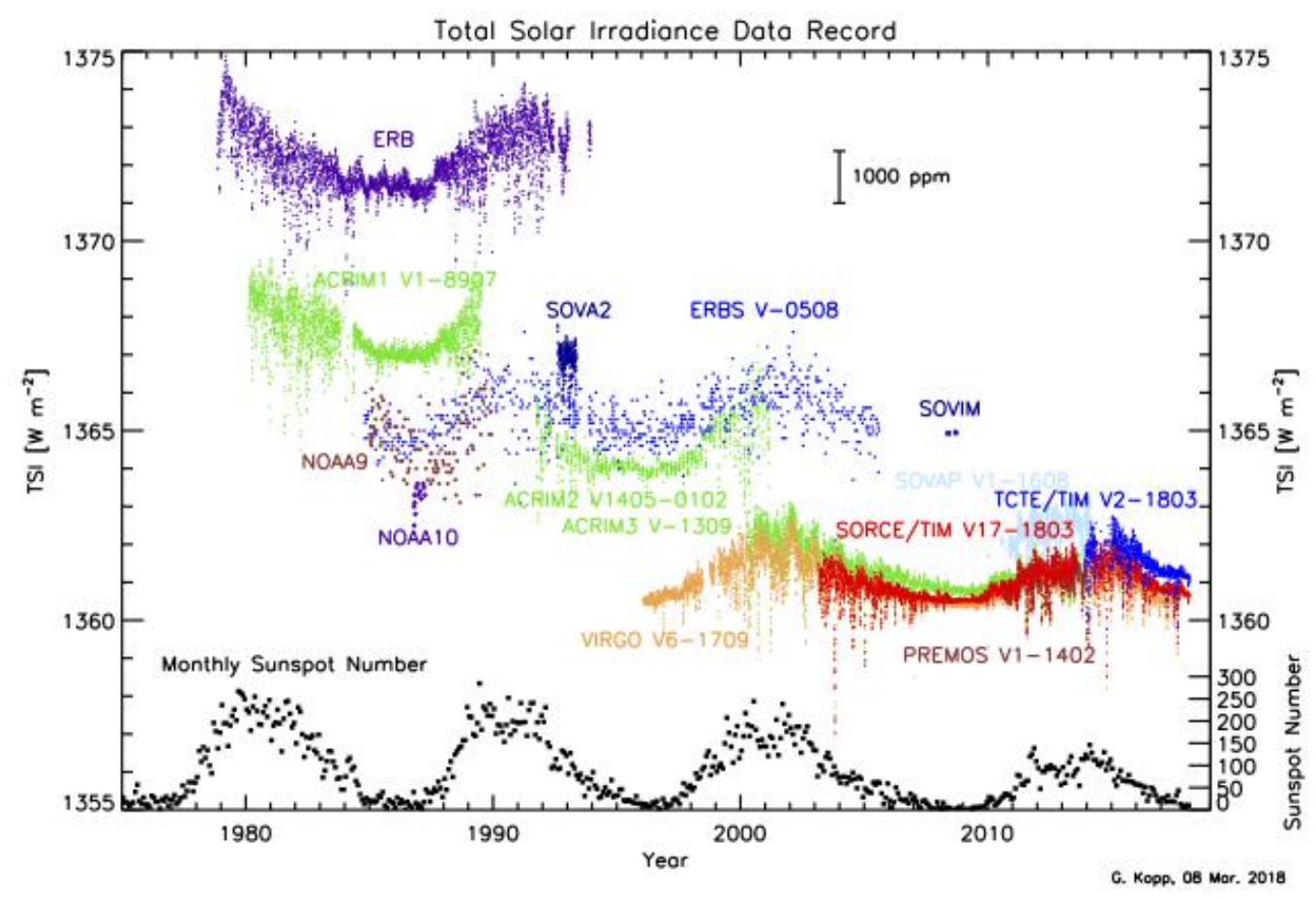

Fig 1. Historical TSI data record [2]

\section{SORCE Spacecraft Overview}

The spacecraft bus is based on the Orbital ATK LEOStar- $2^{\mathrm{TM}}$ spacecraft bus. It is the first redundant version of the LEOStar-2 with only a few single string elements (single battery with extra cells).

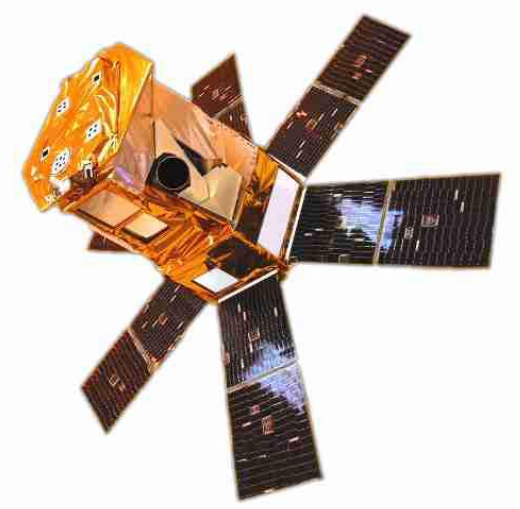

Figure 2. Photograph of SORCE (with background removed)

Figure 2 is an actual photograph (background removed) of what SORCE looks like in its fully deployed on orbit configuration [3]. The spacecraft bus is a hexagonal structure with six deployable solar arrays. The majority of the spacecraft components are installed within the spacecraft structure. The instruments are mounted to the upper portion of the structure with co-aligned boresights along the $+\mathrm{Z}$ axis. During nominal solar operations, the solar arrays and instrument boresights are pointed directly at the sun. The spacecraft performs several rolls about the $\mathrm{Z}$ axis during orbit day to prevent the star trackers from being occulted by the Earth. At night, the spacecraft slews to point at different inertial targets to calibrate the instruments and to prevent the star trackers from becoming occulted by the Earth. This sequence of events is illustrated in Figure 3 and represents the operations concept prior to the battery anomaly. 

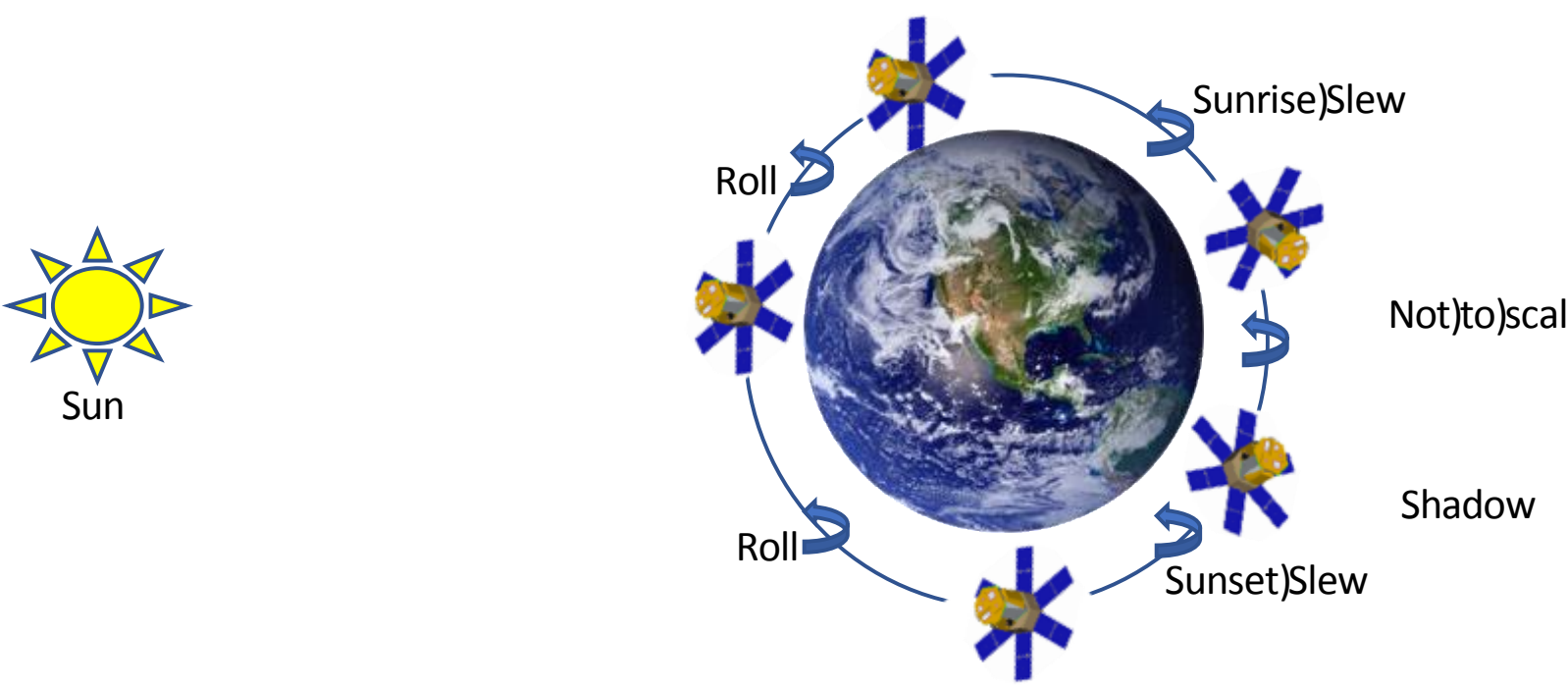

Fig 3. Operations concept detailing solar pointing during orbit day and inertial pointing in eclipse

A brief introduction to key subsystems and the original operations concept are required in order to understand the anomaly, and some of the challenges associated with the recovery. The descriptions below describe the original functionality of the system at launch. Additional capabilities were added to the system to support the transition to DOOP mode. Those changes will be discussed later.

\section{A. Command and Data Handling Subsystem Overview}

The Command and Data Handling $(\mathrm{C} \& D H)$ system contains the brains of the system. It is responsible for the execution of commands and the collection, storage and distribution of data. Three different computers work together to support flight operations: The Central Electronics Unit (CEU) the Attitude and Power Electronics (APE) and the instrument Microprocessor Unit (MU). The CEU serves as the main computer for nominal flight operations. It supports uplink, downlink and the storage of recorded data. In addition, the CEU contains the On Board Computer (OBC) where the main flight software runs. When operating in normal mode, the $\mathrm{OBC}$ is the primary computer in charge. It hosts the absolute and relative time-tagged commands as well as the sophisticated telemetry monitoring and fault response system. The Attitude Power and Electronics (APE) unit serves as the interface between the OBC and the majority of spacecraft components. In addition, the APE executes a subset of the software installed on the OBC and is capable of controlling the spacecraft in lieu of the OBC. When operating in safemode the APE is the primary computer and is often referred to as the safehold computer. The MU serves as the single interface between the instruments and the spacecraft. All commands and telemetry to and from the instruments are routed through the MU. In addition, the MU has a command sequencing capability independent from the OBC. This allows the MU to store and execute time tagged command sequences for the instruments.

\section{B. Attitude Determination and Control Subsystem Overview}

The Attitude Determination and Control Subsystem (ADCS) is a 3-axis stabilized, zero momentum design. The system includes two star trackers and a fine sun sensor to provide high precision attitude knowledge. It uses four reaction wheels to control where the spacecraft is pointed. Magnetometers and torque rods are used to control the momentum of the system. When not in a science data collection mode, coarse sun sensors are used identify the direction of the sun vector in the body frame during orbit day.

There are three primary operational modes for ADCS: normal mode, contingency mode and safemode. Normal mode represents the highest functioning attitude control mode. In normal mode, the $\mathrm{OBC}$ is controlling the pointing of the spacecraft. In this mode, all attitude sensors are used to precisely point the spacecraft at the commanded target. The spacecraft must be in normal mode to collect quality science data. The second control mode is referred to as contingency mode. The OBC is also in control of pointing the spacecraft in contingency mode. This mode uses a subset of the attitude sensors, primarily coarse sun sensors, to point the spacecraft within a few degrees of the Sun. The third control mode is safemode which is implemented in the APE. Safemode uses the same sensors for input as contingency mode and has very similar attitude performance. 


\section{Thermal Control System Overview}

The thermal control system uses a combination of surface treatments, multi-layer insulation blankets and heaters to maintain components within acceptable limits. When in OBC control, some of the heaters are enabled and disabled based on set points within the software. Heaters which are controlled this way are referred to as the operational heaters. Some heaters are enabled and disabled autonomously by thermostats. These heaters are referred to as the survival heaters. Generally speaking the operational heaters are used to control temperatures when SORCE is in normal mode or contingency mode. SORCE relies on the survival heaters when in safemode.

\section{Telecommunications System Overview}

The spacecraft supports communications through two omni-directional antennas. One antenna is mounted on the $+\mathrm{Z}$-side of the spacecraft with the other mounted on the $-\mathrm{Z}$-side. The two antennas are coupled together providing near spherical coverage for uplink and downlink. The telecommunication system is capable of communicating with ground antennas in Santiago Chile and Wallops Island Virginia as well as NASA's Tracking and Data Relay Satellites (TDRS). Orbital geometry provides four ground station visibilities each day which can be augmented with continuous coverage through TDRS.

\section{E. Fault Protection Overview}

The spacecraft fault protection system is primarily hosted within the OBC. The system is highly configurable and can be set up to monitor any telemetry point on the observatory. When a telemetry threshold is exceeded the fault detection system will initiate the appropriate response by calling a stored command sequence.

\section{F. Electrical Power Subsystem Overview}

The Electrical Power Subsystem (EPS) generates, stores and distributes power throughout the spacecraft. The six solar arrays are in a fixed orientation with respect to the spacecraft body frame. All solar cells are on located on the $+\mathrm{Z}$-side of the panels and are co-aligned with the instrument boresights. Power generated by the arrays is distributed to the various components on the spacecraft and to recharge the battery. Excess power is dissipated through shunts in the power regulation electronics. The battery is composed of 11 Nickel-Hydrogen Common Pressure Vessels (CPVs). Each of the CPVs contain two cells with each cell capable of reaching 1.6 volts when fully charged. The original battery was rated a $23 \mathrm{Ah}$ of capacity.

\section{Mission Operations}

The SORCE mission operations team is composed of a number of small teams with specific areas of responsibility and expertise. The mission management team includes the Principle Investigator, the director of the Mission Operations and Data Systems division at LASP and the NASA mission manager. The Flight Operations Team (FOT) is composed of a mix of LASP professional research assistants (flight controllers) and student operators (command controllers) from the University of Colorado. The FOT supports daily operations of the observatory (spacecraft and instruments). This includes planning and scheduling of activities, state of health monitoring and anomaly resolution. The Engineering support team contains members of the FOT and is augmented with instrument specialists from LASP and engineers from Orbital ATK. The TDRS scheduling team is a subset of the FOT. This team is composed of specially trained student operators. These students are responsible for generating the access times between SORCE and the TDRS constellation and submitting requests for individual supports (200+ supports per week). In addition, the TDRS team responds to change requests from the White Sands scheduling office. During anomalies the FOT, Engineering support team, TDRS scheduling team and mission management teams work together as the Anomaly Resolution Team (ART). The Mission Operations Manager (MOM) directs the daily activities to investigate the anomaly and to support the return to science. The MOM works to keep the management team informed of progress and provides recommendations to management for approval when necessary. Figure 4 summarizes each of the different teams. 


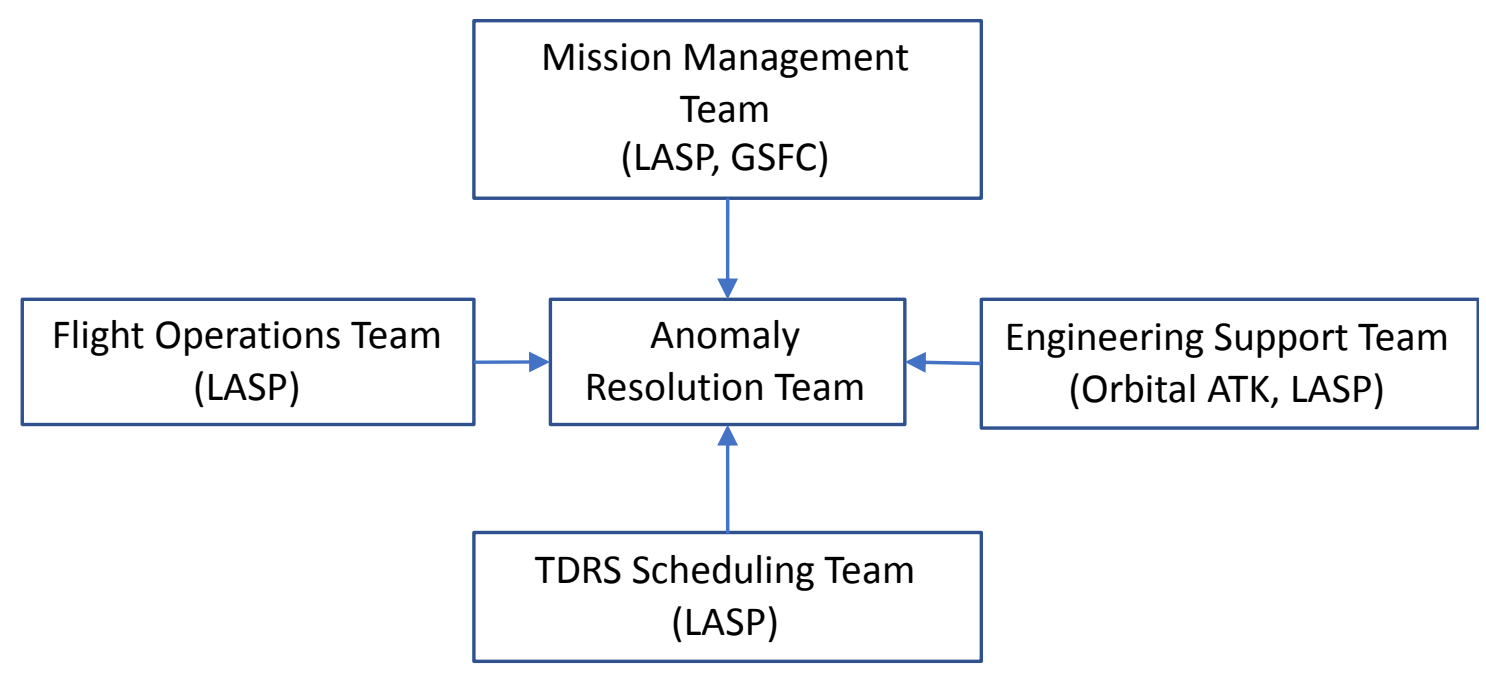

Fig 4. SORCE mission operations support teams

The SORCE Mission Operations Center (MOC) is located in the multi-mission operations facility at LASP. In routine operations the core of the FOT consists of four professionals and a number of graduate and undergraduate students. SORCE and Aeronomy of Ice in the Mesosphere missions are based on similar Orbital ATK architectures and as a result the same FOT is used to support both missions. This cross-staffing arrangement provides financial benefits to both programs while ensuring that lessons learned from one program are passed on to the other. In the event of an anomaly the LASP FOT and engineering teams can be supported from the MOC. As needed, real-time and recorded telemetry can be made available to the Orbital ATK team located in Sterling VA. Having the ability to forward real-time data to the engineering team was very useful during the recovery activities. This remote viewing capability allowed the Orbital ATK to fully participate in the recovery process while eliminating down time since no travel from one facility to the other was required. In addition, it allowed the key members of the Orbital ATK team to remain co-located during recovery activities.

SORCE was launched into a $640 \mathrm{~km}$ circular orbit inclined at 40 degrees. In this orbit the spacecraft completes one orbit of the Earth every 96 minutes. Due to the orbit geometry, each orbit will experience an eclipse that varies from 12 to 35.5 minutes in duration. The variability of this eclipse can be seen in Figure 5 and offers unique challenges and opportunities for the FOT. Several key events in the recovery timeline are included in Figure 5. 


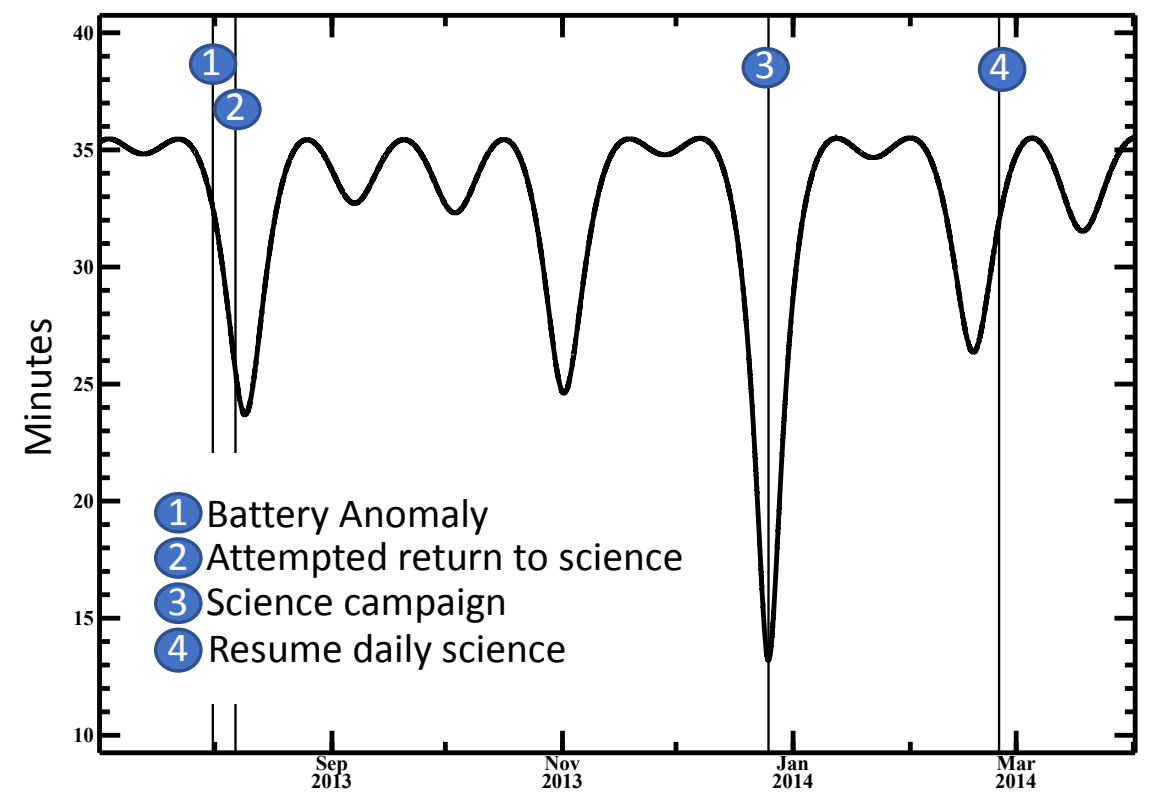

Fig 5. Eclipse durations seen by the SORCE spacecraft during the recovery process including key milestones

Prior to the events in the second half of 2013, a number of cells within the battery were demonstrating significantly reduced performance. Specifically, five of the twenty-two cells in the battery would fully discharge in eclipse. The operations team had taken many steps to reduce the number of loads powered in eclipse. This included turning off the entire suite of instruments each sunset and turning them back on each sunrise. Similarly, the spacecraft was configured to its lowest possible power mode in eclipse while remaining in normal mode. In this configuration, the APE, OBC, star tracker and reaction wheels as well as other non-switchable critical loads were powered throughout the orbit.

In June of 2013, a new flight software image was loaded to the OBC. This activity required the operations team to develop a plan for how to safely and efficiently command the spacecraft to safehold, operate in a "normal" safehold configuration while loading the new software, and transition back to normal mode.

On July $15^{\text {th }} 2013$, an attitude control anomaly resulted in poor solar pointing. As a consequence of the poor pointing, the system experienced a deep discharge of the battery. During this event a sixth cell within the battery failed and another started to show abnormal behavior. Both of these issues further reduced the capacity of the battery. The engineering teams from LASP and Orbital ATK worked together to generate command sequences to return the spacecraft to normal operations. Within thirty hours of the initial anomaly, the spacecraft had been reconfigured and returned to normal science operations. This recovery sequence was made significantly easier due to the re-use of scripts generated to support the software load in June.

The operations team continued to closely monitor the performance of the additional weak cell in the battery. Onboard fault detection and correction set points and responses were updated to provide a more aggressive response to further reductions in battery capacity. At the time, it was unclear if the loss of another cell would result in a mission ending event.

The events leading up to the anomaly are important due to the fact that they required the operations team to exercise the process of getting into and out of safemode efficiently. This increased team familiarity with that process and allowed the team to identify improvements that could be capitalized on in future recovery operations. 


\section{SORCE Battery Anomaly}

On July $30^{\text {th }}, 2013$, the weakened battery cell failed causing the bus voltage to fall low enough that the OBC was turned off and the spacecraft regressed to safemode. This represented the lowest voltage seen by the spacecraft since launch. It is unknown how low the voltage was due to the fact that the recorded information was lost when the OBC was powered off. Based on the telemetry available, it appeared that the OBC had been switched off just prior to sunrise. The eclipse duration at this time was 33 minutes and getting shorter (identified as event \#1 in Figure 5). The operations team quickly transitioned into a $24 \times 7$ staffing mode to support the spacecraft while in safemode.

\section{A. Phase 1: Stabilize the system and transition to a robust and simplified operations concept}

The plan at the time of the anomaly was to leave the spacecraft in safemode if an under-voltage event occurred. Accordingly, the goal of the first day was to transition to a safemode configuration which used the minimum power in eclipse. On the first orbit the non-operational/spare reaction wheel and the star tracker were powered off. In addition, the transmitter and $\mathrm{OBC}$ would be turned off prior to sunset and would then be commanded back on by the FOT at the next sunrise.

The ART was quickly convened to review spacecraft status and approve the plan going forward. The next priority was to manually control the battery heaters. Normally in safehold the battery temperature is controlled by survival heaters which are activated by thermostats with fixed set points. Relying on thermostats to keep the battery warm was undesirable as it would allow the battery to get colder than desired and more importantly, the heaters could turn on in eclipse increasing the battery depth of discharge. A stored command sequence was developed that would turn on the battery heater for several minutes as needed. The FOT monitored the battery temperature in real-time and if the battery temperature was low, the battery heater sequence would be loaded and executed.

The final goal of day one was to turn the operational heaters back on and improve the reliability of getting loads turned off prior to sunset. For the first four orbits the FOT was dependent upon having the ability to command the spacecraft near sunset to get the $\mathrm{OBC}$ and transmitter turned off. This required a successful contact with the spacecraft either through the ground or space networks. If the team was unable to turn those components off, it was possible that the voltage would be low enough to brownout the OBC and APE leaving the spacecraft in an uncontrolled state. To reduce the likelihood of this happening, a stored command sequence was loaded and executed each orbit. This sequence would initially turn on operational heaters and then prior to sunset command them, the $\mathrm{OBC}$ and the transmitter off. The goal of turning on the operational heaters each orbit was to perform an eclipse preheat to prevent the spacecraft from getting cold and having survival heaters turn on in eclipse. This enhancement also significantly reduced the stress on the FOT. It gave them several opportunities each orbit to load the safing sequence and once it was executing the team only had to monitor the performance for the rest of the orbit. A common theme thorough out the extended anomaly period was to find ways to reduce the likelihood of an operator error and to improve the reliability of the operational sequence. Over the next several orbits additional loads were added to the turn on/off command sequence. The FOT was provided with a suite of command sequences of variable duration that would allow them load and execute the appropriate sequence for the time remaining before sunset. This completed Phase 1 .

\section{Operations Challenge: Backflips}

One of the challenges with operating the spacecraft in safemode or contingency mode is dealing with spacecraft backflips at sunrise. Backflips will occur if the spacecraft drifts too far off the Sun during eclipse. When this happens, the spacecraft detects that the Sun is on the back side of the spacecraft, it maneuvers to an anti-solar attitude and the performs a 180 degree slew to flip the spacecraft back to a solar pointing attitude. Unfortunately, this sequence is undesirable from a power perspective. It effectively extends the eclipse period by increasing the time that the solar array is not generating power, and then it commands a large torque on the reaction wheels during the slew back to solar pointing. Both of these events increase the depth of discharge on the battery bringing the system that much closer to a brownout event. Compounding the problem is the fact that reaction wheels have less torque authority when operated at low voltages. This can cause the backflips to take longer or fail altogether (bad backflip). On average, a backflip would extend the eclipse period by seven minutes on average. A bad backflip could extend the eclipse period to twenty-five minutes or more. It is worth noting that the spacecraft was now being operated at voltages lower than were ever tested pre-launch.

\section{Operations Challenge: Staffing}

Early on in the anomaly it was clear that this event could result in a prolonged period of $24 \times 7$ operations which would require the FOT to be on console almost continuously. As with many programs in the extended mission phase, the size of the operations team had been reduced to a level that was adequate to support routine operations and minor anomalies. It was clear that the existing operations staff would not be adequate to both sustain the operations cadence and work on developing plans and products for future activities. 
After the first two days, the SORCE Flight Controllers (FC) were split into two teams. One team was assigned to support the anomaly resolution process, this was made up of the more experienced FCs, now called the planning team. They focused on anomaly investigation and planning and scheduling of future activities. The second team was responsible for executing on-console operations and training additional staff to support the on-console operations. FCs from other LASP managed missions were identified to assist with SORCE operations. These new FCs were quickly trained in the basics of SORCE operations and then were placed into the shift rotation. Within a few days three new operators were trained to assist with console operations. The console operators were assigned eight hour shifts in a four-day on, four-day off rotation. Given the non-nominal state of the spacecraft, it was desirable to not leave the newly trained operators on their own to deal with new situations as the arose. As a result, the planning team also provided $24 \times 7$ coverage. This was accomplished by converting one of the offices to a dormitory room complete with a bed. It was important for those who had to stay overnight to have a comfortable place outside of the operations area where they could get some rest.

\section{B. Phase 2: Attempt to return to normal operations}

Given then unpredictable performance associated with backflips while in safemode, it was desirable to attempt to return to normal operations. The orbital geometry was such that on August $9^{\text {th }}(10$ days after the anomaly started) the spacecraft would be at a local minimum eclipse duration of 23.5 minutes (event \#2 in Figure 5). This was also the shortest eclipse period for the next four months. Given past battery performance, there was a good chance that the failed cell had recovered and if it had, normal science operations could resume. Before recovery could be attempted, it was necessary to update a couple of settings within the safehold computer to create a more power efficient transition into safemode.

Engineers at Orbital ATK quickly generated, tested and delivered a software patch to update the safehold entry sequence. This patch improved safehold entry by: leaving reaction wheel 3 powered off, not turning on the transmitter, and commanding off the star tracker. Each of these would reduce the battery depth of discharge in the event the spacecraft regressed back to safemode.

Using the products created during the June safemode as a baseline, updated products were created, and on August $6^{\text {th }}$ the FOT attempted to return the spacecraft back to normal operations mode (eclipse duration $=24.5$ minutes). The FOT was able to get the spacecraft fully configured and back into normal operations for one orbit. Unfortunately, the battery voltage was low enough that the FOT could see some hardware based under voltage protection circuits start to activate. It was clear the spacecraft would not be able to remain in normal mode under these conditions and the decision was made to command the vehicle back to safemode.

Lesson Learned \#1: Maintenance of contingency recovery scripts is important. While the team was unsuccessful in being able to keep the spacecraft in normal mode due to battery performance, it was possible to make the attempt because the sequence had recently been exercised (June). If the FOT had to build the entire sequence from scratch it was unlikely that it would have been ready in time to support the opportunity. It is likely that management would have evaluated the risk as being too high.

Lesson Learned \#2 It is important to understand the impact of software changes on routine and contingency operations procedures. After detecting the lower than expected voltage and tripping under voltage protection circuits, the team quickly decided that it was best to return the vehicle back into safemode. Unfortunately, the command sequence used was not the most efficient. This caused the spacecraft to point away from the Sun at the next sunrise. The FOT was initially unable to regain contact with the spacecraft using the expedited acquisition sequence that had been used for the last several days. The expedited sequence worked well for the previous safemode event but did not work this time due to the updated software installed just prior to the event. As a result, it was realized the importance of reviewing the impacts of a software change on routine and contingency procedures. Contact with the spacecraft was restored once the contingency acquisition sequence was uplinked.

While poor battery performance prevented the team from keeping the spacecraft in normal mode, the process of trying to return the spacecraft to normal operations was in no way a failure. The team learned a number of important things during this activity. The team was able to see the performance of the battery in discharge and could now use that information to model the battery performance going forward. Remember that when the spacecraft had the battery issue at the end of July the data from the event was lost when the OBC was turned off. The FOT now had very valuable information that could be used to assess the health of the battery. The team was able to verify that the spacecraft could successfully operate down to a very low voltage. This information was used to plan the next science campaign. 


\section{Phase 3: Plan for a long-term anomaly}

Given that the battery was no longer able to support the pre-anomaly operations concept, the team reprioritized its efforts towards keeping the spacecraft operating, with the goal of resuming science operations during the December 2013 minimum eclipse of 13.2 minutes. The data collected during the August $6^{\text {th }}$ return to science activity indicated that if the spacecraft could remain operational until December, it was likely that science observations could resume for a couple of days. The new key mission objective for the program was to provide simultaneous observations between the SORCE-TIM and the TCTE-TIM. The TCTE-TIM, hosted on the Space Test Program spacecraft known as the STP-Sat3 mission, was due to launch in November with science observations starting December 15th. There was very strong interest within the scientific community to make sure this overlap was successful. Achieving this goal was a driver for many decisions over the next several months.

The ART reconvened to discuss plans going forward. A list of potential improvements to the system was created and the Orbital ATK team was tasked with investigating the feasibility of each of the proposals. In parallel, the FOT refined the requirements for several of the possible improvements. Each of the updates were then evaluated based on several criteria:

Difficulty of implementation - how long would it take to implement and validate

Performance improvement - would the update significantly improve the performance of the system

Risk - would the improvement reduce the risk in the current operations concept, or would it potentially increase the risk by introducing a new capability.

The various options were presented to the Mission Management Team. The decision was made to implement changes that would significantly reduce the risk of the current operations concept and which could be deployed quickly. It was also decided that investigation should continue into some other performance improvements, but they would not be included in the next software build. In many cases these improvements could be accomplished by modifying various parameters in the flight software and would not require a second build of the software to implement.

The new version of safehold software included the following enhancements:

- Incorporate all changes to performance tables that had been made since launch. This would make the FSW match current performance.

- Add the capability to report the minimum bus voltage in eclipse. This would provide the FOT with some insight into the performance of the battery in eclipse.

- Add the capability to autonomously turn on and off components based on a solar array current threshold. This would reduce the risk associated with commanding components on each sunrise and then having to load a command sequence to turn them off at sunset. It would allow the FOT to discontinue 24x7 console operations, and it would reduce the required TDRS scheduled time each orbit.

- Add autonomous control of the battery heaters. This would make it possible to more accurately manage the battery thermal environment which would maximize battery performance.

The team from Orbital ATK proceeded to create a new version of APE software which added these capabilities. In parallel, additional resource were made available to assess the performance of some of the proposed changes.

During this time, the FOT worked on a number of tasks to improve the operability of the ground system. New tools were developed to aid in the selection and scheduling of TDRS contacts. These tools incorporated spacecraft to TDRS geometry to select contacts which avoided SORCE antenna nulls. Additional tools were developed to display spacecraft telemetry as a function of time since sunrise. This allowed operators to easily compare trends from one orbit to the next and to make better selections of which battery heater sequence to run. Finally, automated data processing software was released to properly time tag OBC data from the beginning of each orbit. Prior to this, spacecraft data would be time-stamped with the GPS epoch (January 6, 1980) and would have to be manually corrected to current time by ground operators. This labor-intensive process did not always happen in a timely manner. The updated data processing software provided the operators greater visibility into spacecraft performance as the OBC was booting up.

\section{Phase 4: Implementing high priority improvements}

On September $9^{\text {th }}$, the new version of safehold software was approved and ready for upload to the vehicle. It would take several orbits to complete the load due to the size and the number of real-time activities to be commanded. The first orbit of flight software loads proceeded with no problems. At the start of the next orbit, the operations team was unable to acquire a signal from the spacecraft. The program then declared a spacecraft emergency to ensure that it would be able to get additional TDRS time if needed. For the next eight orbits the FOT worked through the same command sequence for acquiring telemetry that it had been using since the anomaly began. The belief was that the spacecraft had experienced some sort of attitude anomaly caused by the transition from contingency mode back into 
safemode which then resulted in a bad backflip and possibly ended with the spacecraft stuck in a non-solar pointing orientation. The FOT started working through various contingency and fault isolation procedures to try and determine what action to take. During this process the current acquisition sequence was compared to an older contingency sequence. It was noted that the old contingency sequence would command on both of the transmitter switches while the current acquisition sequence would only enable one of them. The missing command was identified and was issued to the spacecraft. The FOT immediately received telemetry from the spacecraft.

Discussing the situation with FOT team members after the event, the team was focused on what they believed the worst-case scenario was - loss of attitude stability resulting in degraded power generation and a possible mission ending event. They then put their efforts towards developing recovery processes for responding to that potential anomaly assuming that they may get only one chance to fix the problem once contact was restored. As a result, the team did not focus on ruling out other potential faults. In addition, the team was not convinced the older fault isolation procedure for telecom outages was up-to-date for the new mode of operations. Consequently, the team initially ignored this sequence as it was deemed un-reliable. While it is true that the fault isolation procedure was in need of some updates for the current spacecraft configuration some of the initial steps in the procedure were still appropriate. Once the FOT started to work through the fault isolation procedure communication with the spacecraft was restored within two orbits. The distraction of pursuing the "worst-case" concern, significantly delayed the recovery. The FOT captured a couple of lessons learned out of this event.

Lesson Learned \#2b: Understanding impacts of flight software changes (repeat). The reason the FOT was unable to establish communication with the spacecraft following the entry in safemode was once again related to the flight software patch that had been loaded in August. When the issue occurred in August, the team solved the problem by running a contingency script associated with being in a high telemetry rate. In this case, the failure was the same, but the team had to recover using the low downlink rate version of the contingency script.

Lesson Learned \#3 Working through fault trees and contingencies is critical during anomalous events. During both communication outages (August $6^{\text {th }}$ and September $9^{\text {th }}$ ), the team was distracted by the concern that instabilities in the ADCS system may lead to a mission ending event. The team focused on that as the most likely problem and did not move on to pursuing other possible failures for several orbits.

Lesson Learned \#4 Importance of a negative acquisition sequence. Trying to restore communication with a spacecraft in an anomalous configuration can be challenging. Operations teams are often working at a disadvantage in that they generally have little to no information to work with. In this case, if the operations team had an up to date, pre-canned fault isolation and recovery procedure with built in criteria for taking more aggressive responses, that could have been quickly deployed with limited resources.

The FOT resumed the APE software load after standing down for two days to focus on review of recovery procedures. Keeping in tune with the old adage "when it rains it pours" the transition to using the updated software was delayed for several days due to the 100-year flood hitting in Boulder county. The MOC remained staffed and operational throughout the event. However, concerns over the reliability of power, ability of staff to reach the facility and the inability of the University to respond to non-life-threatening emergencies warranted a delay of several days before resuming operations.

The deployment of the new APE software added significant capabilities to the system. The software incorporated a number of changes since launch to improve the performance of the ADCS system. Most of these changes were associated with improving the reliability of the backflip maneuver. Having this software available in EEProm significantly reduced the complexity and time to recover from an APE reset.

In addition to ADCS performance updates, this software added new capabilities to the spacecraft. The safehold computer was given the ability to turn hardware on and off based on the amount of power generated by the solar array. Once the spacecraft was solar pointed and generating sufficient power, the APE would turn on additional spacecraft hardware. At sunset, low solar array current, the spacecraft would turn off the unnecessary loads. The system was designed to be table driven so the FOT could enable or disable loads as needed to support special operations. In addition, the new software was able to record the minimum bus voltage during eclipse and telemeter that value to the ground during the next solar period. This update gave the FOT some insight into the performance of the battery. Finally, the new software was able to autonomously control the battery temperature. If the battery was getting too cold, then the heaters would be commanded on. Once the battery reached the desired temperature, the heater would be turned back off. 
With these changes the spacecraft was back to a configuration where under normal conditions it would be able to take care of itself. It was no longer dependent on having the FOT on console each orbit to issue commands to keep it safe. Enabling the new APE software was a major milestone on the road to recovery for the mission. An important benefit of this new software was that it significantly reduced the stress of the operations team. After a couple of weeks of monitoring and trending of minimum bus voltages, the FOT started to gain confidence that SORCE would remain operational long enough to complete the scientific overlap with the TCTE.

Having achieved a more stable operations concept through flight software, additional automated capabilities were added to the ground system. One of the simple yet surprisingly effective updates was to enable audible alarms within the ground monitoring system to advise the team as to the health of the spacecraft each orbit. Prior this improvement, operators would break their chain of thought each spacecraft sunrise and return to the command and control consoles to check on the spacecraft health. These monitoring activities were significantly impacting the productivity of the planning team who were co-located in the operations area so that they were immediately available to the operators if needed. Not only did it pull staff away from other important jobs and distract them from the task at hand, it put them in a very stressful situation multiple times per day. Waiting each sunrise for the spacecraft to wake up and communicate with the ground was incredibly draining on the team. The fact that the spacecraft continued to routinely exit eclipse with the solar arrays pointed away from the sun causing the acquisition telemetry to be delayed by $5-10$ minutes. Having the audible alarms in place combined with the new FSW that would turn on components reduced stress on the team and increased team productivity.

Lesson Learned \#5 The working environment is very important to team productivity and morale. Early in the recovery process the team was not aware as to how much their productivity was being impacted by being in a location where they were monitoring sunrise activities multiple times per day. It is believed that had the recovery team been re-located to a different room near to the operations area that stress would have decreased and productivity would have increased. This impact is hard to quantify however teams working extended recovery efforts should assess the working environment and consider making changes.

Lessons Learned \#6 Multiple command streams with a scripting capability in the MOC command and control system made it easier to implement automation. The SORCE MOC uses Operations and Science Instrument Support - Command and Control (OASIS-CC) as its command and control system. The FOT was able to make use of the parallel command streams to build a robust and automated system. Each of the different command processors could be tasked to perform different functions. Some would monitor telemetry and issue notifications to the FOT, others were dedicated to setting up and tearing down of the system for each contact while others would uplink commands to the spacecraft. All of these activities were running in parallel with watch dog type scripts to monitor the status of the other streams and restart them as necessary.

With the new flight software capabilities and additional telemetry on spacecraft health, the FOT was able to enhance the capabilities of the ground system to respond appropriately to various situations. The FOT monitored the new automated systems for several days, and finally on September $30^{\text {th }}$, the FOT returned to lights-out operations. The FOT remained on site for several more weeks however, they were not required to be on console for each contact. The staffing plan was updated to make sure someone was within the facility and able to respond to alarms from the automated system at each sunrise, but operators were allowed to return to their normal daily routine including returning to a normal sleep schedule. On October $16^{\text {th }}$, all onsite staffing requirements were lifted, this officially ended the $24 \times 7$ support period after 78 days.

The operations team continued to make additional enhancements to the system over time. One very helpful new capability was to give the FOT the ability to remotely monitor spacecraft telemetry. This new capability provided the FOT with access to a rebroadcast of the spacecraft telemetry in areas outside of the operations room. Operators were able to access the data stream from their offices, their home and any other place where they had access to the corporate VPN and an internet connection.

Lesson Learned \#7 - Remote viewing of spacecraft telemetry eases the burden on operations teams. Providing the FOT the ability to remotely view telemetry can reduce the stress on operations teams. Without this ability staff were required to spend more time in the office, often waiting to make sure everything was okay at the next sunrise. With this ability, some members were able to go home early and take care of personal business and still be back on line to support spacecraft activities. It also allowed operators on-site to notify off-site staff of issues and get a well-informed second opinion in a timely manner which reduced 
the stress on the on-console staff. Finally, one issue with supporting an extended anomaly with a small staff is team health. The ability to monitor remotely allowed staff that were feeling ill to isolate themselves from the rest of the team and not get other team members sick.

\section{E. Phase 5: Enhancing capabilities of the system}

One final technical issue remained a significant risk to the short and long-term health of the spacecraft: backflips. The FOT continued to monitor the performance of the spacecraft at sunrise. As can be seen in Figure 6, the spacecraft continued to see frequent backflips with some days having a backflip at every sunrise. Thankfully the initial problem with extended backflips (> 20 minutes in duration) had not been seen since August. The FOT team believes that some of the changes made shortly after the anomaly started reduced the likelihood of bad backflips. Unfortunately, due to a lack telemetry in eclipse, the team could not be certain that they had eliminated the possibility of having a bad backflip. Early in the anomaly resolution process the ART identified a possible solution. This solution involved updating the spacecraft to fly with a bias in system momentum. The theory was if the spacecraft system momentum was increased in the solar pointing direction then there would be additional spacecraft gyroscopic stability in eclipse. This momentum would help to keep the spacecraft pointed in the solar direction during eclipse such that the front side of the spacecraft would be pointed in the direction of the sun at sunrise.

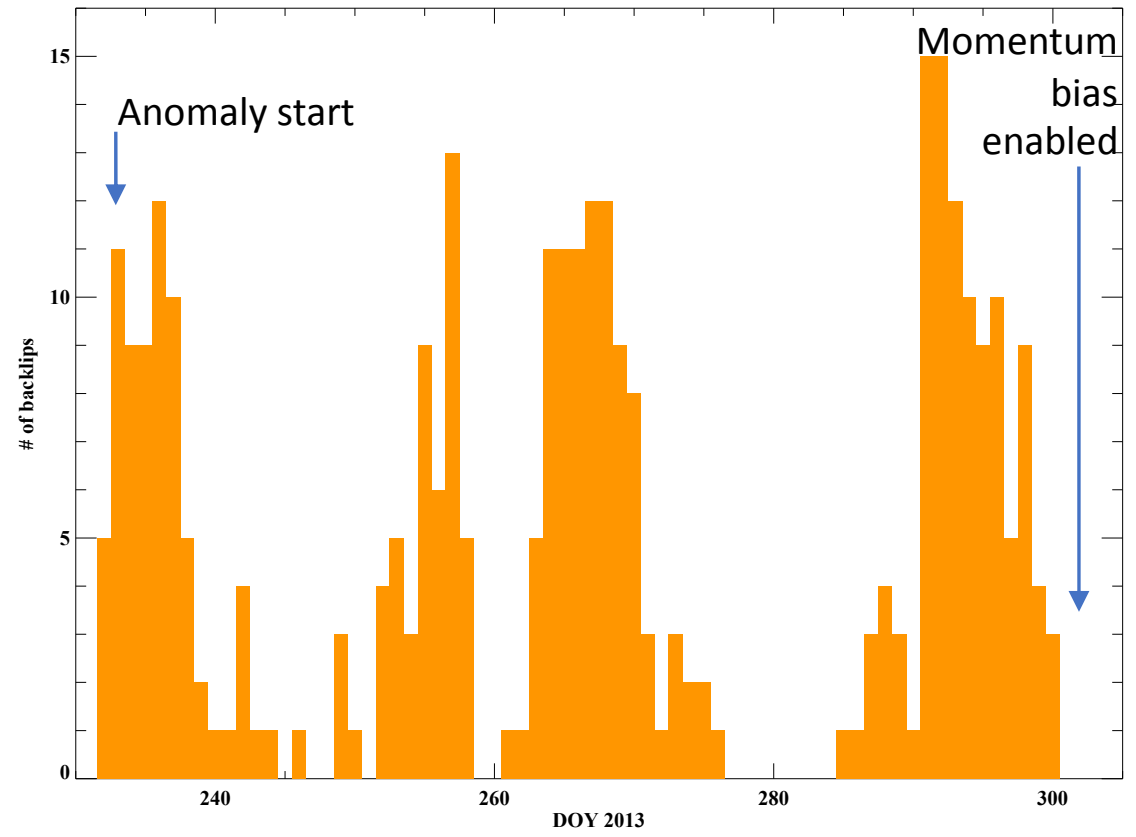

Fig. 6 Number of orbits per day with a backflip slew at sunrise

The concept of using system momentum to stabilize the spacecraft is not new. Many programs have used spinstabilization as an effective attitude control technique. Spacecraft which make use of spin-stabilization however are generally designed and tested to validate their performance prior to launch. The SORCE spacecraft was not designed this way. Fortunately, as can be seen in Figure 2, the spacecraft is fairly symmetric along the axis that is orthogonal to the solar arrays and aligned with the instrument boresights and the desired solar pointing direction. To implement momentum bias mode, the attitude control team at Orbital ATK had to quickly develop and test a new spin-stabilized mode for the spacecraft. Given that this was completely new functionality, the process of building and testing this approach was started early in the recovery process. Fortunately, the ADCS team was able to initially perform software development and testing of the new mode without using flight software resources. This allowed the ADCS team to move forward as the software team was focused on the new APE software.

The engineers at Orbital ATK explored a number of options for how to configure the spacecraft to support a new momentum biased attitude control mode. Final implementation was accomplished by updating tables within the flight software and did not require any code modifications. When operating with momentum bias enabled, the spacecraft stores the extra system momentum in the reaction wheels during orbit day. This allows the instruments to continue to make observations from an inertially fixed attitude. In eclipse, the spacecraft stops sending torque requests to the reaction wheels causing them to spin down. As the reaction wheels spin down, most of the system momentum is transferred from the wheels to the spacecraft body causing the spacecraft to slowly rotate. The system momentum is 
high enough that the induced gyroscopic stability keeps the spacecraft pointed in the direction of the Sun. As the spacecraft exits eclipse, the spacecraft detects the presence of the sun and powers the reaction wheels on. The reaction wheels then absorb the spacecraft body momentum and points at the sun. During orbit day, the spacecraft adjusts reaction wheel speeds to maintain the desired momentum while using magnetic torque bars to remove any extra momentum.

On October 30, 2013, the desired momentum parameters were updated on the spacecraft. An orbit was selected which had a favorable alignment of the body axis and the Earth's magnetic field. Within a single solar period, the spacecraft was able to achieve the desired system momentum. The FOT waited anxiously for the next sunrise to see if the spacecraft performed as expected. The hard work of the team was rewarded with an acquisition of signal at the correct time on the following orbit day. Not only was the spacecraft able to successfully lock onto the sun at sunrise on that orbit but has done so on every orbit since momentum bias has been enabled, over 20,000 orbits. After a week of onsite monitoring, the 24/7 staffing requirement was once again lifted and operations returned to lights-out configuration.

\section{F. Phase 6: Lunar eclipse}

Before the team could completely focus on preparing for the science overlap, there was one more technical challenge to overcome. On November $3^{\text {rd }}$, the Moon would pass in front of the Sun and would cause SORCE to see a reduction in solar intensity on two orbits. Analysis indicated that the intensity would fall low enough to cause the spacecraft to transition into eclipse mode. There was some concern as to how the spacecraft would respond when switching in and out of eclipse mode more than once an orbit. As a result, the FOT updated a number of settings to lower threshold at which the spacecraft will declare sunset. With these changes in place, the spacecraft was able to successfully fly through the events without any impacts to the stability of attitude control system.

\section{The Science Campaign}

Planning for the science overlap campaign had been going on during the months leading up to the event. The updates performed over the previous months had resulted in a spacecraft and ground system that was much more robust to unexpected events and was operating with much more stability. Leading up to the campaign, several checkout activities were performed. The first of which was to verify that the ground-based antennas that are used to capture recorded telemetry were still working as desired. The FOT scheduled several proficiency contacts with the antennas located at Wallops Island, Virginia and Santiago, Chile. These antennas had seen limited use during the recovery efforts. Both stations successfully completed their proficiency contacts and were ready to support the science campaign. The star tracker was turned off in July and had not been powered on for four months. Several days prior to the campaign the star tracker was turned on and a short checkout was performed to verify that it was ready to support the campaign. Finally, new telemetry subscriptions were tested to verify that it was possible to get telemetry data down in real-time through TDRS. This was done to verify that there was a backup capability to capture science data if there were problems getting the recorded data through Wallops or Santiago.

On December 21, 2013, the operations surrounding the science campaign began. The FOT developed a sequence for turning components back on while monitoring battery performance. The plan contained a minimum voltage criterion for proceeding to the next step as well as criteria for terminating observations at the end of the campaign. The first update to the spacecraft was to change the battery charge profile. After the events in August, the FOT switched to using a simple charge control system where the spacecraft battery was significantly overcharged each orbit by applying a fixed charge current throughout the entire solar period. This change in operations concept was required due to unstable performance within the battery which would result in an unpredictable charge profile. As needed, the FOT would command a "charge-hard" sequence where the fixed charge current was raised for a period of several minutes to provide an extra boost to the battery. This was often done to try and improve battery performance in discharge, and to make up for shortened orbit days caused by backflip events near sunrise. Unfortunately, during periods with short eclipse periods, the constant charge current would significantly increase the charge/discharge ratio resulting in elevated battery temps. The higher battery temps would lead to additional stress on the battery and reduced performance in discharge. The FOT developed a strategy to load "charge-easy" sequences where the charge current would be lowered for a timed interval during orbit day. As the eclipse duration decreased, the "charge-easy" sequence would be extended resulting in longer periods of low charge current.

Preparations for the science campaign continued the following day when the star tracker was restored operations. To make sure the data from the star tracker would be accurate, the team started to turn on the star tracker and its heaters during orbit day for several orbits before it was needed. This preconditioning was required to get the star 
tracker temperature stabilized to an operational temperature prior to the start of the campaign. Over the first 24 hours of star tracker operations, several different combinations of heaters were enabled to achieve the desired temperature.

Lesson Learned \#8 Large time critical activities should be broken-up into smaller events. When performing time critical operations, it is important to configure components early to mitigate the risk associated with an unexpected event. For the SORCE some of the checkout activities ended up closer to the actual event than desired leaving little time to work any anomalies that surfaced. For SORCE, the activities surrounding the science campaign were packaged as a single large activity. This required all of the products for the event to be completed before any part was approved for execution. It would have been more efficient to break the events into smaller, more manageable segments. This would have allowed the FOT to approve and execute the checkout activities earlier.

The cadence of activities continued to increase on December 23, 2013 with the first attempts to return to collecting science data. The APE was updated to leave the OBC on throughout the eclipse. Additional ground-based contacts were scheduled so that the FOT could dump the data recorders and monitor the discharge performance of the battery for the first time since July. After confirming that the minimum voltage with the OBC on was high enough for the spacecraft to support additional loads in eclipse, the go ahead was given to exit safehold and to return to science attitude. Unfortunately, when operating in safehold or contingency mode the spacecraft roll axis around the sunline is unconstrained. This means that the single operating star tracker may be occulted by the Earth for significant portions of the orbit. This alignment is especially problematic during the mini-eclipse season. As luck would have it, the FOT had to wait four orbits before the star tracker was not occulted by the Earth. Late in the day on December $23^{\text {rd }}$, the team was able to enter normal mode and resume collecting science during orbit day.

Unfortunately, after two successful orbits of collecting science data, the observatory regressed from normal mode back into contingency mode. At the time, the FOT had limited information as to why the spacecraft had regressed. After verifying that the spacecraft was healthy, the decision was made to try and return to normal mode if the opportunity presented itself. The FOT was aware that there was some risk pushing forward while the investigation into the anomaly continued. With this risk under consideration, several factors played into this decision:

- If the process of returning to normal mode was flawed, and the regression was to occur again there was no indication in the available telemetry available that the spacecraft health was at risk.

- The window for collecting the desired science data was very short.

- The science to be collected was of a very high priority.

- The orbital geometry associated with the mini-eclipse period reduced the likelihood getting into normal mode on any given orbit.

- The team was reaching the end of a very long day and if the spacecraft could not be transitioned back to normal mode soon, then the activity would have to wait several orbits while the FOT rested.

This time the FOT was fortunate and the was able to command SORCE back to normal mode and resume science observations on the first orbit. Unfortunately, at the next sunrise, the spacecraft had once again regressed to contingency mode. Shortly thereafter the FOT identified the problem with the return to science products that was causing the spacecraft to continually regress. At this time the FOT decided that no further attempts to return to science would occur that night due to the need for key members of the team to get some rest. The return to science products were updated, reviewed and approved prior to team leaving for the night.

Lesson Learned \#9 Simulator runs can be very effective in reducing operations errors. In the situation described above, the products used were not tested on the spacecraft simulator prior to execution. However, given the fidelity of the simulator, it is unlikely that the particular error in the sequence would have been caught. Projects should assess the capabilities of the simulators available to support flight product development and strive to have the highest fidelity possible.

On the morning of Dec $24^{\text {th }}$, the FOT returned to finish the transition back into science observing mode. On the first orbit, the FOT found the star tracker unocculted and producing valid quaternions. The team commanded the spacecraft back into normal mode and enabled science data collection with the updated products. On the following sunrise, the team was happy to report that the spacecraft had successfully flown through eclipse without any problems. Over the next several orbits, the FOT closely monitored the minimum voltage in eclipse. Finally, at 22:30 on December $24^{\text {th }}$, the FOT configured the TIM instrument to begin continuous operations. This provided the instrument with the needed temperature stability to complete the cross-calibration with the newly commissioned TCTE-TIM. 
The FOT team continued to monitor the minimum spacecraft voltage in eclipse for the next two days. As the eclipse duration started to increase the minimum voltage started fall. Thirty-seven hours after the TIM was configured to remain on in eclipse, the FOT reconfigured the spacecraft to power off the TIM and instrument computer in eclipse. Day time only science observations continued until December $28^{\text {th }}$ when voltages reached the pre-planned trigger point for returning to safemode. At that point the science campaign was ended and the spacecraft was commanded back to safemode.

Early in the anomaly recovery process the goal of the mission was to make a minimum of 12 hours of continuous TIM measurements with an additional 12 hours of daytime only measurements. At the start of the campaign, the FOT was hopeful that they would be able to collect 24 hours of continuous data with an additional day of data by power cycling in eclipse. The fact that the battery was healthier than expected was a very welcome surprise for the team. It allowed for continuous TIM operations for thirty-seven hours and science to be collected from all six instruments for almost five days. The data collected during this event allowed the science team to complete the cross calibration of the SORCE TIM and TCTE TIM.

\section{Return to daily science observations}

The operations team learned quite a bit during the science campaign as to how the spacecraft would perform during transitions in and out of safemode. Based on the results of the campaign and the consistent battery performance observed since enabling momentum bias, the FOT was optimistic that a new operations concept could be developed that would allow day time only science observations to continue. The science data overlap achieved during the science campaign provided the minimum data necessary to compare the performance of the two TIM instruments. The science team had a strong desire to continue to take orbit day data to collect additional science to establish a longer baseline of joint observations. The science team was extremely interested in comparing the data collected over three full solar rotations ( 72 days). The data collected during the campaign also indicated that all instruments were still healthy after nearly five months in hibernation. As a result, in January 2014 the operations team began ground testing of new operational sequences which would allow the spacecraft to resume daily observations of the sun.

The biggest technical challenge with returning to making daily solar measurements was how to get the data to the ground. The battery was not healthy enough to support leaving the $\mathrm{OBC}$ on in eclipse, as a result data could not be preserved from one orbit to the next. The spacecraft was able to communicate to the ground via TDRS, however, there was only enough bandwidth available to downlink data from one instrument at a time. This limitation would greatly reduce the science return in this mode. The spacecraft team from Orbital ATK reviewed the design of the downlink system and determined that by adding a few commands to the command database, that it would be possible to increase the TDRS data downlink rate form $4 \mathrm{kbps}$ to $8 \mathrm{kbs}$. This additional bandwidth would support the downlinking of all data from all instruments as well as the ancillary spacecraft performance data needed to process the science data.

The SORCE team worked with the engineering team at the TDRS operations facility to define new downlink parameters to allow for the capture of the science data in real-time at the new data rate. On January $16^{\text {th }}$, the new data rates were tested and worked on the very first attempt. This new capability was incorporated into the sequences used to support Daylight Only Operations (DO-OP).

The FOT, Orbital ATK and LASP engineering teams worked together to update SORCE's flight software to incorporate lessons learned from the previous six months of operations. To accomplish all of this work efficiently, the FOT was essentially divided into two teams. One team focused on the changes to the spacecraft while the second team worked on the instrument command sequences. For the updates to the spacecraft, the FOT took the lead on developing new stored command sequences and updating the telemetry monitoring system to trigger the appropriate command sequences each orbit. The Orbital ATK team provided valuable updates and technical input to the sequences proposed by the FOT and eventually incorporated them into a new build of flight software. In parallel to these activities, the second tem worked with the LASP engineering and science teams to update the software running within the instrument microprocessor unit.

Finally, on February $24^{\text {th }} 2014$, DO-OP was enabled operationally and the SORCE resumed making daily measurements of the sun. Since that time SORCE has continued to make solar measurements nearly every day. The operations team has made several updates to DO-OP since it was deployed operationally. These updates improved the robustness of the system, increased the amount of science collected each day and has further reduce the number of loads powered in eclipse. The robustness of DO-OP mode was tested in 2017 when battery performance degraded further and resulted in more than 200 orbits where even the APE would shut down in eclipse, effectively passivating the spacecraft. Within 48 hours of the beginning of this event, the autonomous systems were updated and SORCE returned to making daily solar measurements. 
Lesson Learned \#10 Large, complicated tasks are more efficiently managed by smaller technical teams working on individual problems. Given the complexity of the sequences required to support DO-OP it was very challenging to keep track of all the individual parts. By dividing the tasks into two parts, each team was able to make more progress by focusing on their portion of the problem.

Lessons Learned \#11: Distributed autonomy can be an effective solution for overcoming operational challenges. Due to limitations within the spacecraft design, it was clear that ground commanding would be an important part of the solution. Given that this dependency was acknowledged early, the team could pursue solutions that would leverage the capabilities of the ground system. As a result, this reduced the number and complexity of changes required to flight software.

\section{Conclusion}

The battery anomaly in July was nearly a mission ending event for SORCE. During the recovery process there were several close calls that could have also ended the mission. Through hard work, creativity, incremental improvements and an enormous team effort, the operations teams were able to stabilize the spacecraft, improve the reliability and eventually return SORCE to an operational configuration. In February 2014 NASA authorized the SORCE mission to transition to a new operations concept, DO-OP, and based on the success of this new mode, has approved two additional mission extensions. This has allowed SORCE to complete the cross-calibration of TCTETIM and is now in the process cross calibrating both TSI and SSI measurements with instruments on the Total Solar Irradiance Mission.

The SORCE team approached the extended recovery process in much the same manner as documented in CloudSat Anomaly Recovery and Operational Lessons Learned. The SORCE team shares many of the same observations as documented in the keys to success and team takeaways.

\section{Acknowledgments}

The authors would like to thank NASA for providing the funding and technical support of the SORCE recovery efforts. We sincerely thank our colleagues at GSFC for having confidence in our team and believing that SORCE could be returned to daily science observations. Your support and timely technical advice contributed to our successful recovery. There are several members of the extended NASA family that we would like to thank. Specifically, the team is thankful for the support from the TDRS operators and scheduling team in White Sands NM. The service you provided to the SORCE team was instrumental in the successful recovery of SORCE and the continued success of the mission. In addition, we would like to thank E. Moyer at GSFC for his support throughout. Your words of encouragement and timely technical suggestions was greatly appreciated.

Additionally, the authors would like to thank the engineering support team at Orbital ATK. Your technical recommendations and solutions were key our success. Specifically, we would like to acknowledge the efforts of D. Oberg, G. Baird and A. Wang.

Most importantly we would like to thank all members of the team and their families who supported us during this extended recovery. The missed weekends, holidays and family events were too many to count. We appreciate your passion, dedication and your willingness to make the sacrifices necessary to support SORCE. 


\section{Appendix A}

\section{Acronym List}

$\begin{array}{ll}\text { ADCS } & \text { Attitude Determination and Control Subsystem } \\ \text { Ah } & \text { Amp-hour } \\ \text { APE } & \text { Attitude and Power Electronics } \\ \text { ART } & \text { Anomaly Resolution Team } \\ \text { C\&DH } & \text { Command and Data Handling } \\ \text { CEU } & \text { Central Electronics Unit } \\ \text { CPV } & \text { Common Pressure Vessels } \\ \text { DO-OP } & \text { Daylight Only Operations } \\ \text { DSMC } & \text { Data Services Management Center } \\ \text { EOS } & \text { Earth Observing System } \\ \text { EPS } & \text { Electrical Power Subsystem } \\ \text { FC } & \text { Flight Controllers } \\ \text { FOT } & \text { Flight Operations Team } \\ \text { GSFC } & \text { Goddard Space Flight Center } \\ \text { km } & \text { Kilometers } \\ \text { LASP } & \text { Laboratory for Atmospheric and Space Physics } \\ \text { MOC } & \text { Mission Operations Center } \\ \text { MOM } & \text { Mission Operations Manager } \\ \text { MU } & \text { Microprocessor Unit } \\ \text { OASIS-CC } & \text { Operations and Science Instrument Support - Command and Control } \\ \text { OSC } & \text { Orbital Sciences Corporation } \\ \text { SORCE } & \text { Solar Radiation and Climate Experiment } \\ \text { SSI } & \text { Spectral Solar Irradiance } \\ \text { TCTE } & \text { TSI Calibration Transfer Mission } \\ \text { TDRS } & \text { Tracking and Data Relay Satellites } \\ \text { TSI } & \text { Total Solar Irradiance }\end{array}$




\section{References}

[1] Rottman, G., "The SORCE Mission”, Solar Physics, 230, 1 Aug 2005, pp.7-25

[2] Kopp, G, "TSI Climate Data Record” http://spot.colorado.edu/ koppg/TSI/", March 2018, Accessed: 26 March 2018.

[3] Sparn, T., Rottman, G., Woods, T., Boyle, B., Kohnert, R., Ryan, S., Davis, R., Fulton, R., Ochs, W., “The SORCE spacecraft and operations", Solar Physics, 230, 1 Aug 2005, pp. 71-89 